\title{
Human rights and legal dimensions of self care interventions for sexual and reproductive health
}

\author{
Understanding the user, the health system, and the environment is key to ensuring that self care \\ interventions for sexual and reproductive health are not only available but safe and empowering \\ for all, say Laura Ferguson and colleagues
}

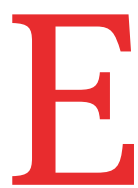

volving technologies are increasing the availability of interventions for sexual and reproductive health that are traditionally offered only in health facilities. Self care interventions for sexual and reproductive health are diverse and include self injection (eg, of contraceptives), self screening or testing (eg, for sexually transmitted infections (STIs) and pregnancy), self medication (eg, for abortion and HIV), and self monitoring (eg, of fertility).

Self care interventions are already in use around the globe and are expanding rapidly with minimal regulation or guidance. Although they can improve access for hard-to-reach populations and reduce the burden on overstretched health services, the potential for misuse and possible harms for users must be considered. The benefits, risks, rights, and health concerns will vary by self care intervention, health system, legal context, and characteristics of the user. When appropriate safeguards are in place, self care interventions can contribute to improving rights and health; when information, support, and quality control are lacking, the reverse may occur.

A human rights and legal analysis can highlight potential concerns and help

\section{KEY MESSAGES}

- The potential benefits of self care interventions must be balanced against the harms, including human rights violations

- Self care interventions should be introduced only when adequate health system support is available and should be an option not the only means of access

- Attention may be needed in places where the law limits access to self care interventions

- A human rights analysis can identify actions to ensure safe and effective provision of self care interventions for all potential users inform the enabling environment required to promote appropriate and equal access to quality self care interventions, especially for people in situations of vulnerability.

\section{Conceptual framework}

A broad range of human rights are relevant to promoting and protecting sexual and reproductive health and rights, but some are recognised to be particularly important. ${ }^{1-5}$ These rights can form a conceptual framework for assessing the human rights dimensions of self care interventions (box 1).

\section{Overarching considerations}

Human rights and legal dimensions vary by product, type of self care intervention, where the intervention is procured (eg, pharmacy, clinic, hospital, online, or shop), and where it is used. For some self care interventions, such as self injectable contraceptives, users still need to see a health worker or pharmacist to obtain them (at least initially). That some of these products can be bought over the counter or online raises complex legal, regulatory, and policy challenges for the user, health system, and responsible regulatory channels. Extrapolating the human rights and legal questions necessitates a clear understanding of the broader context affecting access and use of individual self care interventions in different places, as well as the different groups of users who might be particularly vulnerable to potential adverse effects. This can help determine how each intervention can con-

Box 1: Main human rights implicated in self care interventions for sexual and reproductive health

- The right to the highest attainable standard of health-For the user, ability to engage in self care interventions that are available, accessible, acceptable, and of good quality is key. From the perspective of the duty bearer (usually a state actor who has an obligation to respect, protect, and fulfil human rights), this should form the basis of laws, policies, and regulations governing self care interventions

- Active and fully informed participation of individuals in how self care interventions are rolled out. This supports other relevant rights, including informed decision making, privacy, and confidentiality

- Non-discrimination-Highlights the challenges faced by people who may be marginalised or face discrimination and stigma when accessing services because of, for example, their gender, race, sexual orientation, ethnicity, or ability

- The right to seek, receive, and impart information-This relates to how the provision of information is regulated, including where liability falls for inaccurate or false information. It is particularly important for self care interventions for which users must seek information themselves, often relying on publicly available information rather than health professionals to make appropriate self care decisions

- Informed decision making-For self care interventions this is shaped by whether government actors, manufacturers, service providers, or others facilitate such decision making, including through provision of information that is accurate, accessible, clear, user friendly, and nondiscriminatory

- Privacy and confidentiality are important for access, use, and results of self care interventions. Within the formal healthcare system, there is generally some degree of adherence to medical and human rights standards of privacy and confidentiality. When self care interventions are accessed online or in other non-medical settings, such guarantees may require further consideration

- Accountability-The human rights and legal dimensions of accountability in relation to self care interventions encompass the health sector as a whole, regulation of the private sector, the legal and policy environment more broadly, and access to a system of redress 
tribute to enhanced health, autonomy, and empowerment as well as potential burdens.

Assessment of the legal environment, including laws criminalising certain behaviours or fostering discrimination, can help identify approaches that can ensure safe access to and use of self care interventions. Functional mechanisms such as judicial and administrative modalities are needed to ensure requisite protections and responses to potential rights violations.

Individuals using self care interventions benefit from some connection with the health system to enable appropriate support, including deciding whether to use the intervention, ensuring that they understand how to use it, and access to health professionals for follow-up as required. ${ }^{6}$ This requires that users of self care interventions and healthcare providers are given appropriate information, as well as ensuring availability, accessibility, acceptability, and quality of the services.

A key concern is that self care interventions may contribute to individuals becoming disconnected from health providers. A clear distinction exists between people using self care interventions who know they can connect to a strong health system if required and people using self care interventions because the health system is not available to them or does not meet their needs. Self care interventions must be an adjunct to, rather than a replacement for, interaction with the health system. To be safe, effective, and available to people who are hard to reach, a self care intervention may need more, not less, support from the health system. For example, different ways have to be devised to provide information through non-traditional channels to populations with diverse needs, including different levels of literacy.

Regulation of self care interventions should comply with human rights law and obligations with sensitivity to the

\section{Box 2: Self administration of hormones for gender affirmation}

\section{Right to highest attainable standard of health}

The use of hormones for gender affirmation has generally been found to improve psychological health and relieve physical discomfort and distress among trans people. ${ }^{9}$ If hormone use for gender affirmation is not covered by health insurance, people may be unable to exercise their right to health. A study in New York City found that $23 \%$ of trans hormone users accessed hormones outside the formal healthcare setting from a nonphysician. $^{10}$

Even in countries where formal medical systems encompass quality hormone therapy for gender affirmation, it is not always accessible, acceptable, or affordable, and services often do not use gender neutral language or meet the multiple healthcare needs of trans people. ${ }^{11}$ In many countries, trans people are required to receive a diagnosis of mental illness and undergo psychological or psychiatric assessment ${ }^{1213}$ before accessing gender affirming care, which violates their rights. Such marginalisation can cause people to access hormones without prescription, risking purchasing misnamed or impure hormones and adverse effects that can lead to serious complications. ${ }^{14}$

\section{Participation}

In countries that criminalise trans gender identity and expression, hormones for gender affirmation may be available only outside the formal healthcare system, presenting a substantial barrier to use. Where hormone treatment is available, users may still be reluctant to access formal healthcare because of stigma, discrimination, and lack of legal protection for trans rights. One study noted users were pressured to undergo gender affirming health procedures or testing for sexually transmitted infections, including HIV. ${ }^{11}$

\section{Non-discrimination}

Studies show that trans and gender non-conforming people face discrimination and poor treatment in healthcare settings, creating serious barriers to care. For example, in Malaysia where healthcare is free in the public system, a study found that trans people were mistreated, denied care, or asked to present as a specific gender when trying to use government clinics. ${ }^{11}$ A survey in Ontario, Canada, found that " $43.9 \%$ of trans and gender non-conforming individuals experienced inequalities in the access to healthcare and remained medically unsupervised." ${ }^{14}$ Some trans people may face additional discrimination because of their race, ethnicity, age, and location that further hampers safe and affordable access and use of quality hormones.

\section{Information}

Purchasing hormones online, on the streets, or even in pharmacies without a prescription is associated with having less information about the risks and side effects of treatment ${ }^{1415}$

\section{Informed decision making}

Individuals seeking hormones for gender affirmation confront numerous barriers to accessing the information that would enable them to make fully informed decisions. Inadequate knowledge and potential discriminatory attitudes can affect health workers' willingness to discuss hormone use with trans people or those who are going through gender affirmation. This might encourage people to obtain hormones elsewhere. Informed decision making is likely inhibited further in environments where trans identity is criminalised, leaving few options for self education.

\section{Privacy and confidentiality}

Issues of privacy and confidentiality are connected to the question of legality as legal barriers to accessing services may compel people to seek hormones outside the health system. Unprescribed hormone use, especially purchase in public spaces, puts privacy and confidentiality at risk. People selling or distributing hormones outside the health system are not held to the standards of privacy and confidentiality generally required of health workers by law.

\section{Accountability}

Barriers to ensuring accountability exist where purchase and use of hormones take place in an unregulated setting. Those selling or distributing hormones are not held to drug quality standards or medical training generally required within health systems, leaving users vulnerable to violations without legal redress. In settings where there is no regulation to protect trans and gender non-conforming people or these populations are criminalised, there may be no mechanisms for legal redress if their rights are violated. 
differences across interventions, users, and locations in which interventions are purchased and used. It is critical to balance ensuring quality and safety against access. For example, in places with informal, unregulated markets for medical abortion, the drive to regulate this self care intervention to ensure safety and quality must consider the implications of affordability and accessibility for populations who face discrimination and may be afraid to access health services. It is important to consider how such populations might be identified and how regulations can be tailored to ensure their needs are taken into account in different locations and in relation to different interventions.

An in-depth analysis of the diverse social, economic, and political contexts of individuals and communities, as well as the potential benefits and harms for different users, is critical to creating an appropriate enabling environment for all self care interventions for sexual and reproductive health. Some self care interventions may be expected to be reasonably safe and may increase access without imposing additional burdens on the health system (eg, in specific circumstances, the WHO recommends the self administration of medical abortion pills without direct supervision of a healthcare provider ${ }^{7}$ ), whereas others are potentially more harmful, especially in the absence of appropriate linkage to services (eg, self testing for HIV without access to counselling which has, in some instances, been found to impede uptake of confirmatory testing as well as relevant care and treatment services. ${ }^{8}$

To dig deeper into the human rights aspects of self care interventions, boxes 2 and 3 analyse the human rights dimensions of self injection of hormones for two different purposes and within very different legal and social contexts. The first is unprescribed (medically unmonitored) hormone treatment for gender affirmation; the second is self administration of prescribed hormone treatment for assisted reproduction.

In both of these situations, users want to be able to self inject hormones safely and with appropriate information and support. Our analysis, based on the framework in box 1, highlights existing shortcomings inside and outside the health system, drawing attention to the need to provide information about the self care intervention to users in language they can understand, to promote affordable access to the intervention without discrimination through or with links to the formal health system, and to create avenues for accountability where rights may be violated. Beyond an adequate legal environment, this may also require reshaping health services, including the

\section{Box 3: Self administration of hormones in assisted reproduction}

\section{Right to the highest attainable standard of health}

Use of hormones for assisted reproduction carries psychological benefits but can also lead to substantial stress if use does not result in pregnancy or leads to medical complications. ${ }^{16}$ Use of these hormones is almost solely within the context of the health system, and there can be legal protections that promote rights. Assisted reproduction is often not covered by health insurance, and can be unaffordable and inaccessible for many.

\section{Participation}

Participation in assisted reproduction services can often take place openly. Where infertility remains a source of stigma, women may choose to access services covertly. Participation may be constrained by ability to pay as well as adherence to gender norms; $44 \%$ of those surveyed in a global International Federation of Fertility Societies study indicated couples need to be "in a recognized or stable heterosexual relationship" to access assisted reproduction services. ${ }^{7}$ Only 14 countries reported that assisted reproduction services were available for trans or intersex people. ${ }^{17}$

\section{Non-discrimination}

Many countries with health plan coverage for infertility treatments have age restrictions on access. ${ }^{17}$ Except where serious taboos around infertility remain, people using hormones for assisted reproduction are often socially accepted, perhaps even valourised. However, access is generally easier for those who most closely align to the heteronormative child bearing couple- racial-ethnic majority, able bodied, heterosexual, and in a permanent partnership. Some countries' laws limit access to assisted reproduction, often barring single women, women in same sex relationships, and trans people. ${ }^{17}$

\section{Information}

The use of hormones for assisted reproduction tends to be initiated in healthcare settings so information is relatively accessible, at least for people who can read the dominant language of their country. The availability of information in non-written formats and non-primary languages may be limited. Those who do not access hormones within the formal health system may not have access to information. ${ }^{18}$

\section{Informed decision making}

Some health practitioners may not provide accurate and accessible information about the stress of using hormones to assist reproduction, negative effects of such hormone use, or the associated costs. Informed decision making may be inhibited if providers are biased against single women, women in same sex relationships, and women with disabilities, even where protective laws exist, ${ }^{1920}$ or if health practitioners have financial interests in providing assisted reproduction.

\section{Privacy and confidentiality}

For those accessing assisted reproduction within the context of the health system, regulations usually exist to safeguard privacy and confidentiality. Outside the health system, this may require specific attention.

\section{Accountability}

Regulation of assisted reproduction varies widely, with many countries lacking any formal legislation or regulatory structures. ${ }^{17}$ Even in countries with formal legislation, mechanisms for legal redress may not exist and the penalty varies widely. ${ }^{17}$ Regulations which restrict access for specific populations on the basis of gender identity, sexual orientation, or marital status may not be recognised as discrimination, and these populations are not protected by law. Barriers to ensuring accountability may exist for anyone trying to access assisted reproduction services in countries without anti-discriminatory policies or formal assisted reproduction regulation. 
training of health workers and expansion of insurance schemes.

\section{Implications for rolling out self care interventions}

Assessing and ensuring an enabling environment in which self care interventions can be made available safely and appropriately must be the cornerstone of any strategy to introduce or expand use of these interventions. In countries with a dysfunctional public health system or a weakly regulated powerful private sector, for example, there is high risk that self care interventions for sexual and reproductive health may reach users without the necessary checks and balances to assure their rights and health. The conceptual framework used in our examples systematically draws attention to potential human rights concerns and can inform actions that might reduce the risks.

This type of analysis could be used to inform the implementation of any self care intervention, although the concerns raised may differ from those highlighted in our examples. The more specific the situation analysed, the more targeted the emerging recommendations for action can be. Ensuring broad participation of users, providers, and others can help promote the inclusion of appropriate perspectives to ensure relevance and traction of identified actions.

\section{Conclusion}

No self care intervention functions in a neutral environment or can be considered a neutral activity. The potential of these interventions to increase autonomy, empowerment, and health must be assessed against the potential for unintended consequences, which may vary widely across contexts and communities. Human rights provide an invaluable framework for ensuring self care interventions are safe and effective for all potential users.

We thank Lucinda O'Hanlon, Steven de la Torre, and Kristin Dessie Zacharias for their help with background research, review, and referencing for this article.

Contributors and sources: This article builds on work commissioned by WHO to inform an expert meeting on user initiated interventions for sexual and reproductive health and rights held in March 2018. LF, SG, and STF contributed to a relevant background paper for the meeting, and LF and SR attended the meeting. LF had the idea for the article and it was drafted by STF and LF. SG, SR and TM provided substantive input. All authors have substantial experience working on sexual and reproductive health and rights and gender in different countries and types of institutions. All authors approved the final version submitted. LF is the guarantor.

Competing interests: We have read and understood BMJ policy on declaration of interests and have no relevant interests to declare.

Provenance and peer review: Commissioned; externally peer reviewed.

This article is part of a series proposed by the UNDP/ UNFPA/Unicef/WHO/World Bank Special Programme for Human Reproduction (HRP) and commissioned by The BMJ. The BMJ retained full editorial control over external peer review, editing, and publication of these articles. Open access fees are funded by HRP.

Laura Ferguson, assistant professor ${ }^{1}$

Susana Fried, visiting fellow ${ }^{2}$

Thabo Matsaseng, head ${ }^{3}$

Sundari Ravindran, professor ${ }^{4}$

Sofia Gruskin, professor $^{1}$

${ }^{1}$ Keck School of Medicine of the University of Southern California, Institute on Inequalities in Global Health, Los Angeles, California, USA

${ }^{2}$ Yale Law School, Global Health Justice Partnership, New Haven, Connecticut, USA

${ }^{3}$ Reproductive Medicine Unit, Stellenbosch University, Stellenbosch, Western Cape, South Africa

${ }^{4}$ Sree Chitra Tirunal Institute for Medical Sciences and Technology, Thiruvananthapuram, Kerala, India

Correspondence to: LFerguson

laurafer@usc.edu

\section{(c) (1) (8) OPEN ACCESS}

This is an Open Access article distributed under the terms of the Creative Commons Attribution IGO License (https://creativecommons.org/licenses/ by-nc/3.0/igo/), which permits use, distribution, and reproduction for non-commercial purposes in any medium, provided the original work is properly cited.

\section{Check for updates}

1 WHO. Framework for ensuring human rights in the provision of contraceptive information and services. WHO, 2014.

2 WHO. Ensuring human rights in the provision of contraceptive information and services: guidance and recommendations. WHO, 2014

3 Gender, Human Rights and Culture Branch of the UNFPA Technical Division (GHRCB), Program on International Health and Human Rights, Harvard School of Public Health. A Human Rights-Based Approach to Programming. UNFPA, 2010.

4 Gruskin S, Ahmed S, Bogecho D, et al. Human rights in health systems frameworks: what is there, what is missing and why does it matter?Glob Public Health 2012;7:337-51. doi:10.1080/17441692.20 11.651733

5 UN Committee on Economic, Social and Cultural Rights. General comment No 22 (2016) on the right to sexual and reproductive health (article 12 of the
International Covenant on Economic, Social and Cultural Rights), 2 May 2016. E/C.12/GC/22. UN. 2016.

6 Narasimhan M, Allotey P, Hardon A. Self care interventions to advance health and wellbeing: a conceptual framework to inform normative guidance. BMJ 2019;365:1688. doi:10.1136/bmj.1688

7 WHO. Medical management of abortion. WHO, 2018

8 Wood BR, Ballenger C, Stekler JD. Arguments for and against HIV self-testing. HIV AIDS (Auckl) 2014;6:117-26.

9 White Hughto JM, Reisner SL. A systematic review of the effects of hormone therapy on psychological functioning and quality of life in transgender individuals. Transgend Health 2016;1:21-31. doi:10.1089/trgh.2015.0008

10 Sanchez NF, Sanchez JP, Danoff A. Health care utilization, barriers to care, and hormone usage among male-to-female transgender persons in New York City. Am J Public Health 2009;99:713-9. doi:10.2105/AJPH.2007.132035

11 Gibson BA, Brown SE, Rutledge R, Wickersham JA, Kamarulzaman A, Altice FL. Gender identity, healthcare access, and risk reduction among Malaysia's mak nyah community. Glob Public Health 2016;11:1010-25. doi:10.1080/17441692. 2015.1134614

12 Robles R, Fresán A, Vega-Ramírez H, et al. Removing transgender identity from the classification of mental disorders: a Mexican field study for ICD-11. Lancet Psychiatry 2016;3:850-9. doi:10.1016/S22150366(16)30165-1

13 UN OHCHR. "Pathologization-being lesbian, gay, bisexual and/or trans is not an illness" 17 May 2016 https://www.ohchr.org/EN/NewsEvents/Pages/ DisplayNews.aspx?News|D=19956\&Lang|D=E.

14 Metastasio A, Negri A, Martinotti G, Corazza O.The case of self-prescribing sexual hormones in gender affirmation in individuals attending psychiatric services. Transitioning bodies. Brain Sci 2018;8:E88. doi:10.3390/brainsci8050088

15 Aguayo-Romero RA, Reisen CA, Zea MC, Bianchi FT, Poppen PJ. Gender affirmation and body modification among transgender persons in Bogotá, Colombia. Int J Transgend 2015;16:103-15. doi:10.1080/15532 739.2015.1075930

16 Beutel M, Kupfer J, Kirchmeyer P, et al. Treatment related stresses and depression in couples undergoing assisted reproductive treatment by IVF or ICSI. Andrologia 1999;31:27-35. doi:10.1111/j.1439-0272.1999.tb02839.x

17 Chen X. A new platform for global health research and policy exchange and communication. Glob Health Res Policy 2016;1(e1):1-143. doi:10.1186/ s41256-016-0001-z

18 Elfin DA. Costs, coverage issues spur black market for fertility meds. Bloomberg Law 2018. https://news. bloomberglaw.com/pharma-and-life-sciences/costscoverage-issues-spur-black-market-for-fertility-meds.

19 Klitzman R. "Will they be good enough parents?": Ethical dilemmas, views, and decisions among assisted reproductive technology (ART) providers. AJOB Empir Bioeth 2017;8:253-65. doi:10.1080/23 294515.2017.1394927

20 Lawrence RE, Rasinski KA, Yoon JD, Curlin FA. Obstetrician-gynecologists' beliefs about assisted reproductive technologies. Obstet Gynecol 2010;116:127-35. doi:10.1097/ AOG.0b013e3181e 2 f27d

Cite this as: $B M J$ 2019;365:l1941 http://dx.doi.org/10.1136/bmj.l1941 